\title{
Single-Trial Variable Model for Event-Related fMRI Data Analysis
}

\author{
Yingli Lu, Tianzi Jiang*, Senior Member, IEEE, and Yufeng Zang
}

\begin{abstract}
Most methods for fMRI data analysis assume that the hemodynamic responses (HRs) across similar experimental events are same. This assumption is not appropriate when HRs vary unpredictably from trial to trial. Here, we introduce a new method for fMRI data analysis. The main features of the proposed method are as follows: 1) The trial-to-trial variability is modeled as meaningful signal rather than assuming that the same HR is evoked in each trial; 2) Since the proposed method is a constrained optimization based general framework, it could be extended by utilizing prior knowledge of HR; 3) The traditional deconvolution method can be included into our method as a special case. A comparison of performance on simulated fMRI datasets is made using the general linear model, the deconvolution method and the proposed method with receiver operating characteristic (ROC) methodology. In addition, we examined the effectiveness and usefulness of our method on real experimental data.
\end{abstract}

Index Terms-Brain, deconvolution, functional MRI, general linear model.

\section{INTRODUCTION}

$\mathbf{S}$ INCE the discovery of fMRI, fMRI activation detection has been an active field of research. Various methods have been proposed for analysis of fMRI BOLD datasets. Generally, these can be classified into data-driven and model-driven. Typical data-driven methods are clustering analysis [1]-[4], principal component analysis (PCA) [5], [6], independent component analysis (ICA) [7]-[9], and self-organizing maps (SOMS) [10]. These techniques can separate different types of hemodynamic responses (HRs) without the hypothesis of a paradigm or hemodynamic response function (HRF). The power of these methods is that they can identify nonanticipated or transient task-related components. These methods are complementary to the model-driven approaches.

In practical applications, for the easy of application and physiological explanation, model-driven methods are adopted by many neuroimaging researchers. A typical model-driven method is the general linear model (GLM) [11]. GLM is a framework that includes simple t-test, analysis of variance

\footnotetext{
Manuscript received April 21, 2004; revised October 25, 2004. This work was supported in part by the Natural Science Foundation of China under Grant 30425004 and Grant 601721302, and in part by the National Key Basic Research and Development Program (973) under Grant 2003CB716104. The Associate Editor responsible for coordinating the review of this paper and recommending its publication was P. Thompson. Asterisk indicates corresponding author.

Y. Lu and Y. Zang are with the National Laboratory of Pattern Recognition, Institute of Automation, Chinese Academy of Sciences, Beijing 100080, P. R. China.

T. Jiang is with the National Laboratory of Pattern Recognition, Institute of Automation, Chinese Academy of Sciences, Beijing 100080, P. R. China (e-mail: jiangtz@nlpr.ia.ac.cn).

Digital Object Identifier 10.1109/TMI.2004.840294
}

(ANOVA) and multiple regressions. In GLM, once the design matrix is specified, multiple regressions can be used to estimate the parameters. Statistics for the obtained parameters can be considered to constitute a statistical parameter map. Then a hypothesis test is made under the null hypothesis that the voxel is nonactivated. Recently, the deconvolution method [12], [13] has been proposed for fMRI activation detection. It first estimates the impulse response function(s), which is then convolved with the stimulus paradigm to yield the estimated response. Then, various statistics are calculated to indicate the "goodness" of the fit. Finally, according to a predefined level of statistics, the activated voxels can be identified. The deconvolution method assumes that: 1) HRs differ from voxel to voxel, which is suitable for the properties of the BOLD signal; 2) the same HRs are evoked in different trials. However, experiments have shown that the HR differs from person to person, from day to day, and probably also in different regions of the brain [14]. Furthermore, recent studies have shown that the HR may vary from trial to trial [15].

In summary, the deconvolution method is based on a basic assumption: the HRs are the same across trials, i.e., the trial-totrial variability is considered as noise. Although the deconvolution is widely used for fMRI activation detection, it is suboptimal as a method for robust and sensitive analysis of fMRI time series. When HRs vary from trial to trial, an alternative approach is needed to include the trial-to-trial variability. In this paper, we present a novel method to model the trial-to-trial variability.

The rest of this paper is organized as follows. Section II is devoted to a detailed description of the method. In Section III, results of experiments conducted on simulated and in vivo fMRI data are presented. Discussions and conclusions are presented in Section IV.

\section{ALGORITHM}

The proposed method is divided into three stages: 1) the estimation of the initial HRF (iHRF) for each voxel in the dataset; 2) the estimation of HRFs for each trial in a particular voxel's time series; 3 ) statistical hypothesis test. In stage 1), the HRF of each voxel is estimated by utilizing the deconvolution method. In stage 2), the HRFs for each trial are estimated by utilizing a constrained optimization. In stage 3), whether a particular voxel corresponds to an activated one or noise is determined.

\section{A. Estimation of iHRF for Each Voxel}

Typically, the measured fMRI time series can be thought of as containing three components: 1) signal (HRs component arisen from the experimental stimuli); 2) trend (effects of no interest such as the physiological biorhythms and systematic drifts); 3) 
noise. Then the fMRI time series can be modeled by signal plus trend plus noise, i.e.,

$$
\text { data }=\text { signal }+ \text { trend }+ \text { noise. }
$$

If we assume that the hemodynamic system is a linear one, then the measured time series $z(t)$ for the stimulus time series $f(t)$ should be

$$
z(t)=f(t) \otimes h(t)+\text { trend }+\varphi
$$

$h(t)$ is the impulse response function, $\otimes$ denotes the convolution operation and $\varphi$ is the noise in the measurement. From the viewpoint of fMRI, $h(t)$ is the HRF. An approximate estimation of $h(t)$ can be obtained from the measurements $z(t)$ by utilizing the deconvolution method. In order to elucidate the estimation process, we can rewrite (2) as

$$
\begin{aligned}
Z_{n} & =\sum_{m=0}^{P} f_{n-m} h_{m}+\sum_{i=0}^{I} \beta_{i} n^{i}+\underline{\varphi_{n}} \\
n & =\underline{P, P+1, \ldots, N}, \underline{\underline{-1}}
\end{aligned}
$$

where $\sum_{m=0}^{P} f_{n-m} h_{m}$ is the discrete expression of $f(t) \otimes h(t)$, $\sum_{i=0}^{I} \beta_{i} n^{i}$ is the polynomial trend model, $I$ is the degree of the polynomial, $\varphi_{n} \stackrel{i i d}{\sim}\left(0, \sigma^{2}\right)$. Here, modeling the errors as independent identical distribution variables is a simplification, which will be addressed further in the discussion part of the paper. $P$ is the maximum time lag of HRF and $N$ is the number of data points for the time series of a voxel.

Using matrix notation

$$
\begin{aligned}
Z & =\left[\begin{array}{c}
Z_{P} \\
Z_{P+1} \\
\vdots \\
Z_{N-1}
\end{array}\right] \\
X & =\left[\begin{array}{ccccccc}
f_{P} & \cdots & f_{0} & 1 & P & \cdots & P^{i} \\
f_{p+1} & \cdots & f_{1} & 1 & P+1 & \cdots & (P+1)^{i} \\
\vdots & \vdots & \vdots & \vdots & \vdots & \vdots & \vdots \\
f_{N-1} & \cdots & f_{N-P-1} & 1 & N-1 & \cdots & (N-1)^{i}
\end{array}\right] \\
\beta & =\left[\begin{array}{c}
h_{0} \\
\vdots \\
h_{P} \\
\beta_{0} \\
\beta_{1} \\
\vdots \\
\beta_{i}
\end{array}\right] \\
\varphi & =\left[\begin{array}{c}
\varphi_{p} \\
\varphi_{p-1} \\
\vdots \\
\varphi_{N-1}
\end{array}\right] .
\end{aligned}
$$

Equation (3) can then be written:

$$
Z=X \beta+\varphi
$$

The estimated parameter $\hat{\beta}$ of $\beta$ is

$$
\hat{\beta}=\left(X^{T} X\right)^{-1} X^{T} Z
$$

and the estimated iHRF $\mathbf{h}$ is the first $P$ elements in $\hat{\beta}$, i.e., $\mathbf{h}=$ $\left[h_{0}, h_{1}, \ldots, h_{P}\right]^{T}$.

\section{B. Estimation of HRFs for Each Trial}

Since the estimation of $\beta$ is accomplished by minimizing the error sum of squares between the data and the fit, the estimation of the iHRF $\mathbf{h}$ can be rewritten as a least square optimization

$$
\begin{array}{cl}
\min _{\mathbf{h}} & \|\hat{Z}-\mathbf{h} \otimes f\|_{2}^{2} \\
\text { s.t. } & \mathbf{h} \in R^{P} .
\end{array}
$$

$R^{P}$ is the Euclidean P-space, $\hat{Z}$ is the time series with no trend, i.e., $\hat{Z}=\left[\hat{Z}_{P}, \hat{Z}_{P+1}, \ldots, \hat{Z}_{N-1}\right]^{T}$ and the definition of $\hat{Z}_{n}$ is

$$
\hat{Z}_{n}=Z_{n}-\sum_{i=0}^{I} \beta_{i} n^{i} \quad n=P, P+1, \ldots, N-1 .
$$

Since we assume that the HRFs for each trial are different, by analogy with (6), the new least square optimization can be denoted as

$$
\begin{array}{ll}
\min _{\mathbf{H}_{\mathbf{j}}} & \left\|\hat{Z}-\sum_{j=1}^{J} \mathbf{H}_{\mathbf{j}} \otimes f_{j}\right\|_{2}^{2} \\
\text { s.t. } & \mathbf{H}_{\mathbf{j}} \in R^{P} .
\end{array}
$$

The decision vector $\mathbf{H}_{\mathbf{j}}$ is the HRF for trial $j, f_{j}$ is the single trial stimulus time series, i.e.,

$$
f_{j}(t)= \begin{cases}0, & t \neq j \\ 1, & t=j\end{cases}
$$

(values of $f_{j}(t)=0$ denote the baseline (no stimulus being applied) and values of $f_{j}(t)=1$ represent the presence of stimulus) and $J$ is the total number of trials. The problem is that the solutions $\mathbf{H}_{\mathbf{j}}$ may not be what the neurologist expects. That is, although this optimization problem in (8) can be solved, the optimal solutions $\mathbf{H}_{\mathbf{j}}$ may not have any physiological meaning. Therefore, the constraint in (8) is changed to $\mathbf{H}_{\mathbf{j}} \in N(\mathbf{h}, \varepsilon)$, and the constrained least square optimization in (8) is

$$
\begin{array}{ll}
\min _{\mathbf{H}_{\mathbf{j}}} & \left\|\hat{Z}-\sum_{j=1}^{J} \mathbf{H}_{\mathbf{j}} \otimes f_{j}\right\|_{2}^{2} \\
\text { s.t. } & \mathbf{H}_{\mathbf{j}} \in N(\mathbf{h}, \varepsilon)
\end{array}
$$

where $N(\mathbf{h}, \varepsilon)$ is the neighborhood of $\mathbf{h}$. Mathematically, $N(\mathbf{h}, \varepsilon)$ is the decision space. In a real fMRI experiment, based on the property of BOLD signal, sudden changes in different trials are rather rare. That is, for an activated voxel, the shape and magnitude of $\mathbf{H}_{\mathbf{j}}$ should be close to that of $\mathbf{h}$. This is the 
motivation and rationale why the constraint $\mathbf{H}_{\mathbf{j}} \in N(\mathbf{h}, \varepsilon)$ is included. In practice, the simplest definition of $N(\mathbf{h}, \varepsilon)$ may be

$$
N(\mathbf{h}, \varepsilon)=\left[N\left(h_{0}, \varepsilon\right), N\left(h_{1}, \varepsilon\right), \ldots, N\left(h_{p}, \varepsilon\right)\right]^{T}
$$

where $N\left(h_{m}, \varepsilon\right)=\left[h_{m}-\varepsilon, h_{m}+\varepsilon\right], m=0,1, \ldots, P$. The sketch map of $N(\mathbf{h}, \varepsilon)$ is shown in Fig. 1. Minimizing the objective function in (9) provides us with an optimum set of $\mathbf{H}_{\mathbf{j}}$, $j=1,2, \ldots, J$.

The constrained optimization in (9) is a basic framework. In practical applications, some other constraints should be imposed. For instance, Richter et al. [16] claimed, "in the mental rotation task, the full-width at half-maximum (FWHM) of the peak in the fMRI time series in the parietal lobe was equal to the reaction time (RT)." Based on this result, we consider that the ratio of the FWHM of $\mathbf{H}_{\mathbf{i}}$ to $\mathbf{H}_{\mathbf{j}}$ is equal to the ratio of the RT of trial $i$ to trial $j(i \neq j)$. Mathematically, this relation can be denoted as

$$
\operatorname{FWHM}\left(\mathbf{H}_{\mathbf{i}}\right): \operatorname{FWHM}\left(\mathbf{H}_{\mathbf{j}}\right)=\mathrm{RT}_{i}: \mathrm{RT}_{j}, \quad i \neq j .
$$

$\operatorname{FWHM}\left(\mathbf{H}_{\mathbf{i}}\right)$ is the FWHM of the HRF $\mathbf{H}_{\mathbf{i}}, \mathrm{RT}_{\boldsymbol{i}}$ is the RT of the $i$ th trial. By imposing the constraint of (11) to the general framework in (9), the new optimization problem is

$$
\begin{aligned}
\min _{\mathbf{H}_{\mathbf{j}}} & \left\|\hat{Z}-\sum_{j=1}^{J} \mathbf{H}_{\mathbf{j}} \otimes f_{j}\right\|_{2}^{2} \\
\text { s.t. } & \mathbf{H}_{\mathbf{j}} \in N(\mathbf{h}, \varepsilon) \\
& \\
& \mathrm{FWHM}\left(\mathbf{H}_{\mathbf{i}}\right): \operatorname{FWHM}\left(\mathbf{H}_{\mathbf{j}}\right)=\mathrm{RT}_{i}: \mathrm{RT}_{j}, \\
& i \neq j .
\end{aligned}
$$

In (12), the constraint is composed of two components: 1) random component, i.e., constraint $\mathbf{H}_{\mathbf{j}} \in N(\mathbf{h}, \varepsilon)$; 2) systematic component, i.e., constraint $\operatorname{FWHM}\left(\mathbf{H}_{\mathbf{i}}\right)$ : $\operatorname{FWHM}\left(\mathbf{H}_{\mathbf{j}}\right)=\mathrm{RT}_{i}: \mathrm{RT}_{j}, i \neq j$. These two constraints together constitute the decision space for the objective function $\min _{\mathbf{H}_{\mathbf{j}}}\left\|\hat{Z}-\sum_{j=1}^{J} \mathbf{H}_{\mathbf{j}} \otimes f_{j}\right\|_{2}^{2}$. The role of constraint 1) and 2) are, respectively, to assure the physiological meaning of solutions $\mathbf{H}_{\mathbf{j}}$ and the trial-to-trial variability comes from different RTs for different trials.

Before solving (12), it is worth noting apart from the parietal lobe, the relation in (11) may not be tenable for other brain areas. The reason that we include the constraint of (11) is threefold: 1) to demonstrate the usage of the basic framework in (9);2) by utilizing (12), the neurologists may validate the relation of (11) in other brain areas; 3) as the emphasis of this paper is on the methodology of fMRI activation detection, we leave further neural research as future work. In addition, (12) may only be applied to some event-related paradigms where responses can be measured and the RT is related to brain activity.

In order to solve the above mentioned optimization problem, (12) is transformed to

$$
\begin{array}{ll}
\min _{\mathbf{H}} & \left\|\hat{Z}-\sum_{j=1}^{J} F_{j}(\mathbf{H})\right\|_{2}^{2} \\
\text { s.t. } & \mathbf{H} \in N(\mathbf{h}, \varepsilon)
\end{array}
$$

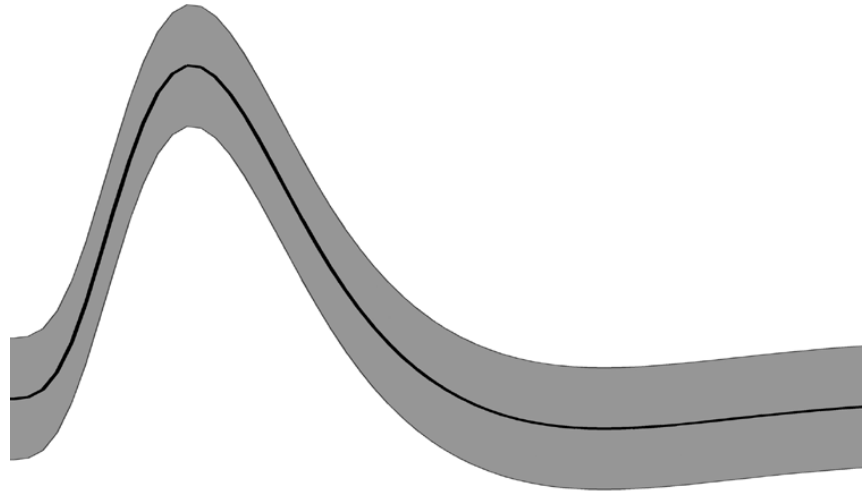

Fig. 1. The strip-like region (gray shading) is the neighborhood $N(\mathbf{h}, \varepsilon)$, $\mathrm{iHRF} \mathbf{h}$ is composed of two gamma functions (black solid line).

where

$$
F_{j}(\mathbf{H})=\operatorname{Interp}\left(\Re_{j}, \mathbf{H},\left[0,1, \ldots, \operatorname{round}\left(\max \left(\Re_{j}\right)\right)\right]^{T}\right)
$$

and

$$
\Re_{j}=\frac{\mathrm{RT}_{j}}{\operatorname{mean}(\mathrm{RT})} \times[0,1,2, \ldots, P]^{T} .
$$

Equation (14) returns vector $F_{j}(\mathbf{H})$ containing elements corresponding to the elements of $\left[0,1, \ldots, \operatorname{round}\left(\max \left(\Re_{j}\right)\right)\right]^{T}$ and determined by interpolation within vectors $\Re_{j}$ and $\mathbf{H}$. The vector $\Re_{j}$ specifies the points at which the data $\mathbf{H}$ is given. $\max \left(\Re_{j}\right)$ is the largest element in vector $\Re_{j}$, and $\operatorname{round}\left(\max \left(\Re_{j}\right)\right)$ rounds the elements of $\max \left(\Re_{j}\right)$ to the nearest integers. mean(RT) is the expected value of the vector $\left[\begin{array}{lll}\mathrm{RT}_{1} & \mathrm{RT}_{2}, \ldots, \mathrm{RT}_{J}\end{array}\right]^{T}$. The essence of (14) is to implement the constraint in (11) by data interpolation. Since $F_{j}(\mathbf{H})$ is what we expect to obtain, the optimum solution $\mathbf{H}_{\mathbf{j}}$, we see that solving for the optimization in (12) reduces to a simple constrained linear least squares optimization in (13). It can be solved easily by an optimization toolbox. In this paper, it is computed by utilizing the Matlab (The MathWorks, Inc.) optimization toolbox.

\section{Statistical Hypothesis Test}

Determining whether a particular voxel's time series corresponds to a signal or not, reduces to the following statistical hypothesis test:

$$
\begin{aligned}
& H_{0}: Z_{n}=\sum_{i=0}^{I} \beta_{i}^{n} i+\varphi_{n} \\
& H_{1}: Z_{n}=\sum_{m=0}^{P} f_{n-m} h_{m}+\sum_{i=0}^{I} \beta_{i n}^{i}+\varphi_{n} .
\end{aligned}
$$

Suppose that $\mathbf{H}_{\min }$ is the optimal solution, then the statistic $F$ is calculated

$$
F=\frac{\frac{S B-S F}{d_{B}-d_{F}}}{\frac{S F}{d_{F}}}
$$

where $\mathrm{SB}=\|\hat{Z}\|_{2}^{2}, \mathrm{SF}=\left\|\hat{Z}-\sum_{j=1}^{J} F_{j}\left(\mathbf{H}_{\min }\right) \otimes f_{j}\right\|_{2}^{2}$, $d_{B}=N-P-2$, and $d_{F}=N-P-2-(P+1)$. SB and SF 
are, respectively, the residual sum of squares of fitting the noise model and the signal plus noise model. $d_{B}$ and $d_{F}$ are, respectively, the number of degrees of freedom for the noise model and signal plus noise model. The statistic $F$ has $F\left(d_{B}-d_{F}, d_{F}\right)$ distribution under the null hypothesis and a larger value for $F$ indicates that the corresponding voxel is activated.

Up to the present time, only a single stimulus function $f(t)$ is considered. However, the extension to multi-input stimuli is straightforward. Suppose that there is another input stimulus $g(t)$, then we have

$$
z(t)=f(t) \otimes h(t)+g(t) \otimes m(t)+\text { trend }+\varphi .
$$

Correspondingly, the basic framework is changed to

$$
\begin{aligned}
\min _{\mathbf{H}_{\mathbf{j}}, \mathbf{M}_{\mathbf{l}}} & \left\|\hat{Z}-\left(\sum_{j=1}^{J} \mathbf{H}_{\mathbf{j}} \otimes f_{j}+\sum_{l=1}^{L} \mathbf{M}_{\mathbf{l}} \otimes g_{l}\right)\right\|_{2}^{2} \\
\text { s.t. } & \mathbf{H}_{\mathbf{j}} \in N(\mathbf{h}, \varepsilon) \\
& \mathbf{M}_{\mathbf{l}} \in N\left(\mathbf{m}, \varepsilon^{\prime}\right) .
\end{aligned}
$$

where $\mathbf{h}$ and $\mathbf{m}$ are the iHRFs of stimuli $f(t)$ and $g(t)$, respectively. It is a multiobjective optimization problem. One formulation for this problem is the goal attainment problem of Gembicki [17] and it can be solved using an optimization toolbox.

\section{EXPERIMENTS AND RESULTS}

In this section, simulated and in vivo data were analyzed by the following three statistical models: 1) the GLM method with parametric modulation [18], [19]; 2) the deconvolution method; and 3) our proposed method. The reason that the GLM and the deconvolution method were chosen for comparison is because they are both popular hypothesis-driven methods for fMRI activation detection.

For the simulated datasets, participants in the fMRI experiments were 8 healthy, right-handed volunteers ( 4 females; age range $21-23$, mean 22 ). We selected randomly one of the 8 subjects (male, 22) as the simulated dataset. For the phonetic decision experiment, 7 native Chinese speakers (4 males and 3 females, $21-32$ yrs) with college or higher education were included. We selected randomly one of the 7 subjects (female, 23) for the comparison of different analysis methods. For both experiments, no patient had a history of neurological or psychiatric disorder. Before subjects were imaged, informed consent was obtained according to the procedure approved by the Institutional Review Board at the Institute of Psychology, Chinese Academy of Sciences.

\section{A. Simulated Event-Related Data}

We used actual baseline data from real fMRI scans instead of computer-simulated ones, since the former more closely match the noise structure of the real fMRI data. Simulated activations were added to the null datasets, as mentioned below. Imaging was acquired on a $1.5 \mathrm{~T}$ scanner SIEMENS Sonata equipped with high-speed gradients. For functional imaging acquisitions, technical parameters were as follows: $2000 / 60 \mathrm{~ms}$ (TR/TE), 20 slices, $64 \times 64$ matrix, $90^{\circ}$ flip angle, 22 -cm field of view, $5-\mathrm{mm}$ section thickness, and 2-mm gap. The null dataset consisted of

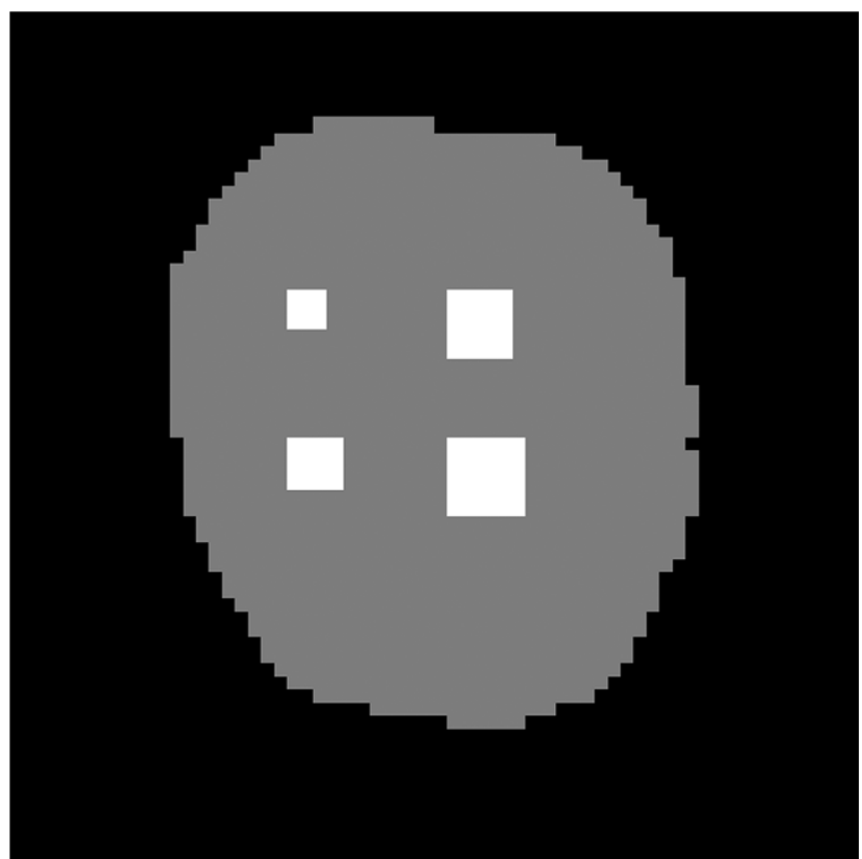

(a)

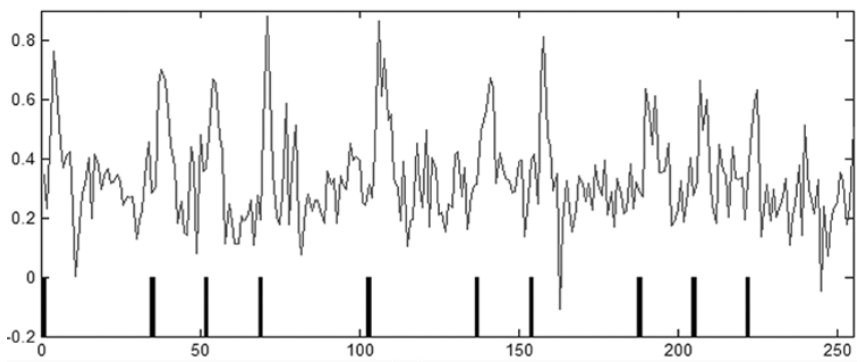

(b)

Fig. 2. (a) One slice of the simulated fMRI data. The white box represents the sites of artificial activated region. (b) The simulated time series of an "activated" voxel.

185 volumes, only the latter 170 volumes were selected. During the acquisition, the subject was instructed to rest, be as motionless as possible, and perform no specific cognitive task.

In this paper, the volume size of the four simulated activated regions corresponded to $3 \times 3 \times 2,4 \times 4 \times 2,5 \times 5 \times 2$, $6 \times 6 \times 2$ cuboids, respectively (refer to Fig. 2). The HRF $\mathbf{H}$ was a combination of two gamma functions [20]. Parameters of the two gamma functions are the defaults of the SPM99 (Wellcome Department of Cognitive Neurology, http://www.fil.ion.ucl.ac.uk/spm) software. The presumed input stimuli $f(t)$ consisted of 170 time points. Eight trials were simulated, and the presumed RT for the ten trials are $\mathrm{RT}=\left[\begin{array}{llllllll}2 & 1 & 0.8 & 1.2 & 1.5 & 1.7 & 0.9 & 0.5\end{array}\right]^{T}$. The simulated activated time series $\sum_{j=1}^{10} F_{j}(\mathbf{H}) \otimes f_{j}$ were then mixed with noise time series from the resting state subject. Eight simulated datasets were generated with the contrast-to-noise ratio (CNR) [21] define as 0.3, 0.6, 0.9, 1.2, 1.5, 1.8, 2.1, 2.4 [22]-[24]. Here, CNR is defined as $a / \sigma$, where $a$ is the amplitude of the normalized ideal waveform and $\sigma$ is the standard deviation of the noise.

The implementation procedure of including parametric modulation in GLM is as follows: 1) take the event times and create 


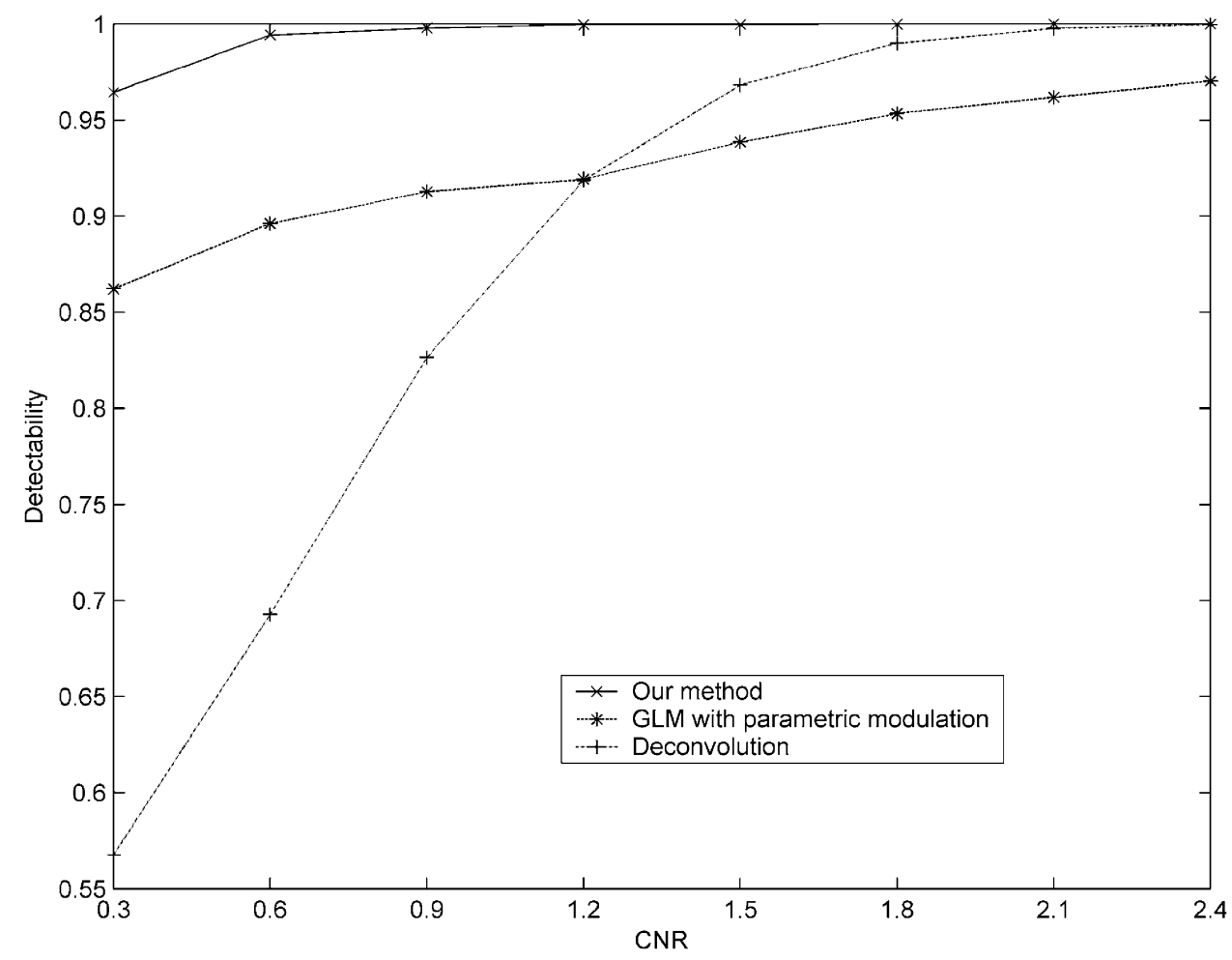

Fig. 3. Performance of our method, the GLM and the deconvolution method for the simulated data.

a set of delta functions, 2) multiply the height of a given delta functions by the RTs, 3) convolve the result of step 2) with the canonical HRF, temporal derivative and dispersion derivative, 4) orthogonalize each term with respect to the zeroth order term [19]. So, the design matrix is set as: the canonical HRF, temporal derivative, dispersion derivative and the corresponding three linear modulated terms.

To give an ROC analysis, four steps were followed: First, the three methods were applied to the simulated datasets. Second, according to the known number of simulated activations, the true positive rate in the activated region and the false positive rate in the nonactivated region were calculated. Third, an ROC curve was calculated by plotting the true positive rate versus the false positive rate. The curve corresponding to a certain method which is closest to the upper left corner should be the best. Fourth, as for the conventional ROC analysis, the area under the ROC curve was taken as the detectability measure of the different methods.

Fig. 3 shows the area under the ROC curve for each of the three methods plotted as a function of CNR. As expected, we find that our method outperforms the other two methods. To further elucidate the increased sensitivity of our method, we examine one particular case, with CNR $=1.5$. The ROC curves of the three methods are depicted in Fig. 4. In Fig. 4(a), the ROC curve of our method approached the top left corner, whereas the ROC curves of the deconvolution method and the GLM method are far from the top left corner. Points $x, y$ and $z$ are the points closest to the optimal classification (false positive rates $=0$, true positive rates $=1$ ) in the respective ROC curves. Fig. 4(b)-(d) illustrates the activation maps obtained with our method, the deconvolution method and the GLM method. The corresponding thresholds were selected according to the value that corresponds to the optimal classification points $x, y$, and $z$. In looking at Fig. 4, it appears that the GLM and the deconvolution method show more activated voxels outside the simulated regions in addition to missing some voxels in the simulated activations. From this comparison, we can conclude that, in the simulated datasets, our method is more sensitive and specific than the other two methods. This is because the FWHM of the HRs is proportional to the width of the RT of each trial whereas, in the GLM model, the FWHM of the HRs is proportional to the height of the RT of each trial. In the deconvolution model, the trial-to-trial variability is considered as noise.

\section{B. In Vivo Event-Related fMRI Data}

This experiment is a study of phonetic decision of visual Chinese characters with a fixed inter-stimulus interval (ISI) eventrelated design. The subject was instructed to press the left button with the left index finger for target stimulus and the right button with the right index finger for nontargets. A $1.5 \mathrm{~T} \mathrm{GE}$ scanner with Echo Planar Imaging (EPI) was used. Fourteen axial slices were acquired with an EPI pulse sequence, parallel to the anterior-posterior commissure (AC-PC) line from about $39 \mathrm{~mm}$ below AC-PC to $59 \mathrm{~mm}$ above it (thickness/gap $=6 / 1 \mathrm{~mm}$, in-plane resolution $=64 \times 64, \mathrm{TR}=2000 \mathrm{~ms}, \mathrm{TE}=40 \mathrm{~ms}$, flip angle $=90^{\circ}$, FOV $\left.=240 \times 240 \mathrm{~mm}\right)$. At the same position, 14 axial slices (thickness/gap $=6 / 1 \mathrm{~mm}$, in-plane resolution $=256 \times 256, \mathrm{FOV}=240 \times 240 \mathrm{~mm}, \mathrm{TR}=500 \mathrm{~ms}$, $\mathrm{TE}=14 \mathrm{~ms}$ ) were acquired as a structural image.

Preprocessing was performed on the in vivo datasets. Images are first realigned with the least squares approach and a 6-parameter rigid transformation in order to reduce the effect of head motion. Then, a spatial smoothing is made to increase the 


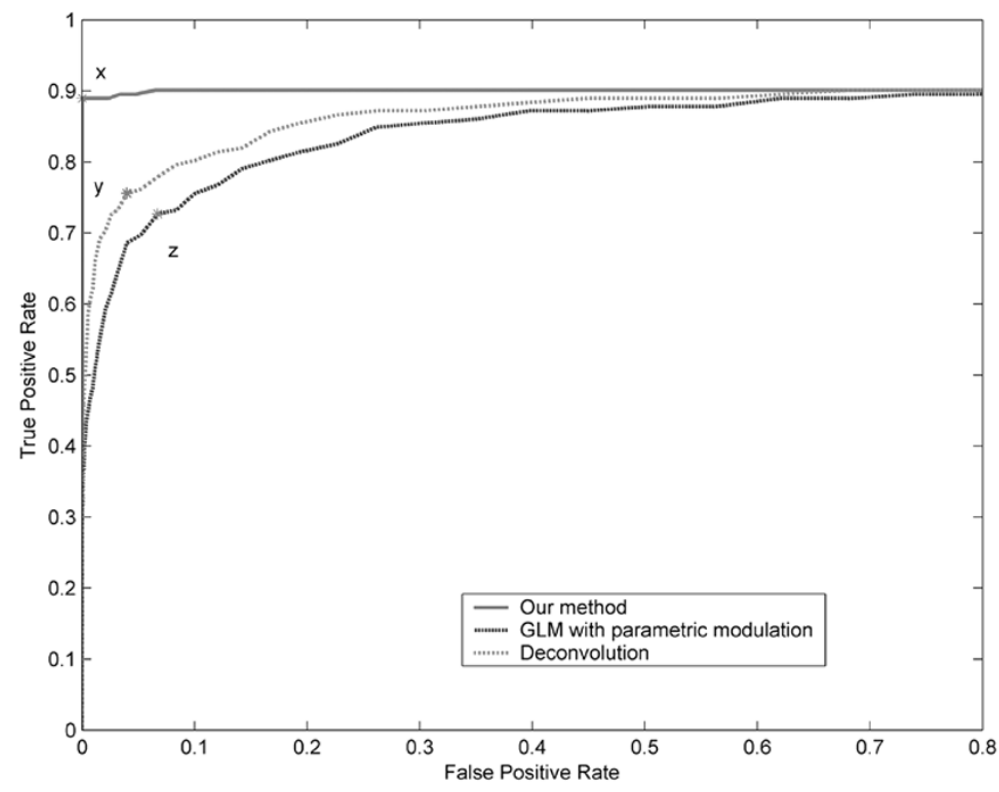

(a)
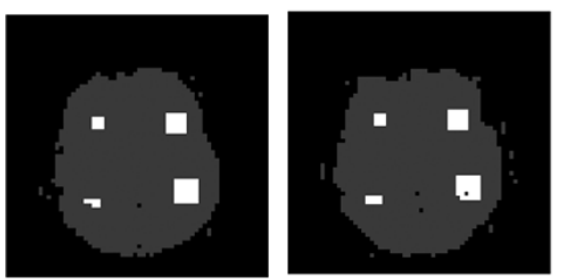

(b)

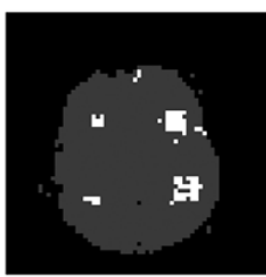

(c)

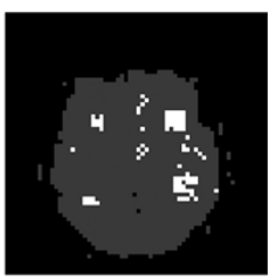

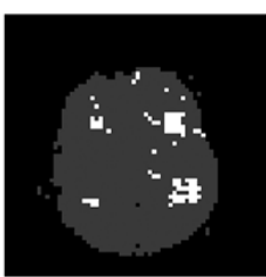

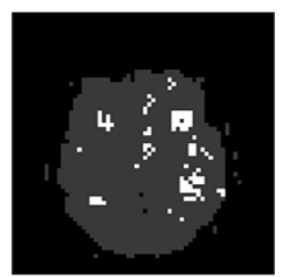

(d)

Fig. 4. (a) ROC curves corresponding to the case of CNR $=1.5$ for the simulated data. (b) Results of our method. (c) Results of the deconvolution method. (d) Results of the GLM method.

signal-to-noise ratio with an isotropic Gaussian filter, in which the FWHM was set to $6 \mathrm{~mm}$. These two procedures were both implemented within the SPM99 software. The dataset was then modeled using the deconvolution and the proposed method. In addition, before doing analysis with the GLM, the dataset was de-trended using the first-order polynomial de-trending method [25].

The design matrix of the GLM with parametric modulation is set as: the canonical HRF, temporal derivative, dispersion derivative and the corresponding three linear modulated terms. For the deconvolution method and the proposed method, the maximum time lag $P$ is set to 7 and the degree of the polynomial $I$ in the baseline model is set to 1 . In addition, for our method, the parameter $\varepsilon$ is empirically set to 2 .

The activation maps are displayed in the form of F-statistic images (for deconvolution and the proposed method) and T-statistic images (for GLM method) overlaid on the structural fMRI images scan from that subject. For the GLM method, only those voxels with $\mathrm{T}$ values above a given threshold of significance $\left(p<10^{-2}\right)$ are shown. For the deconvolution and the proposed method, those voxels with $\mathrm{F}$ values above a given threshold of significance $\left(p<10^{-7}\right)$ are shown. Five important regions that have been reported consistently in the previous studies of the phonetic decision task are the primary motor cortex area (M1), the supplementary motor area, the cerebellum, the fusiform gyrus, and the lingual gyrus. Figs. 5

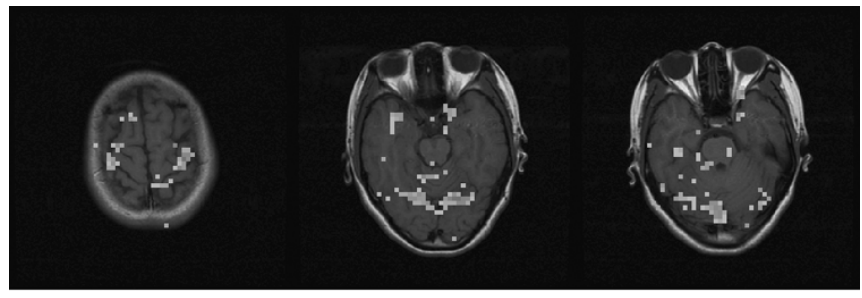

(a)

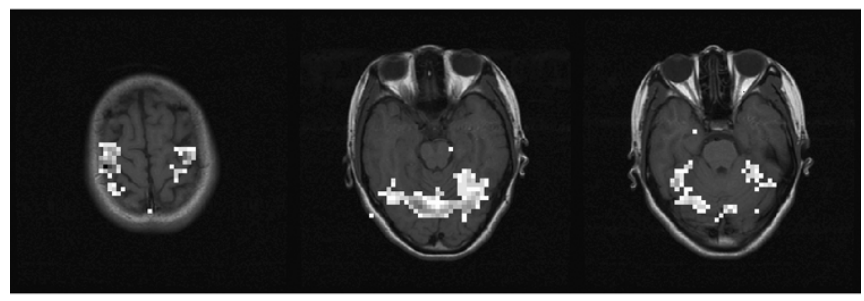

(b)

Fig. 5. Results of comparison for in vivo fMRI data from a PD task (activation maps.) (a) Activation maps of the GLM method $\left(p<10^{-2}\right)$. (b) Activation maps of our method $\left(p<10^{-7}\right)$.

and 6 show just the first and twelfth through thirteenth slices of the total 14 axial slices. One slice is for M1, another is for the fusiform gyrus and lingual gyrus and the other is for the cerebellum. All three methods successfully detected the activation in M1, cerebellum, fusiform gyrus, and lingual gyrus. As the emphasis of this paper is on the methodology of obtaining the 


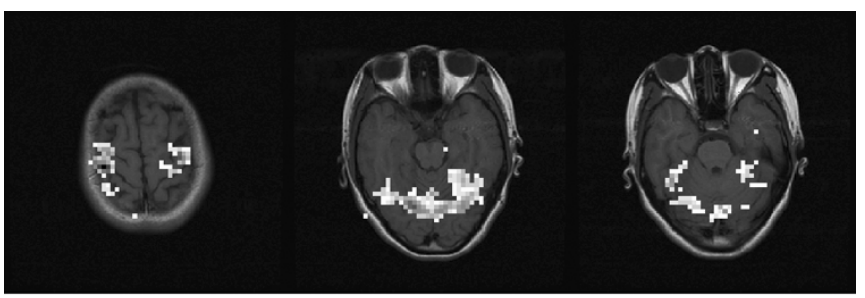

(a)

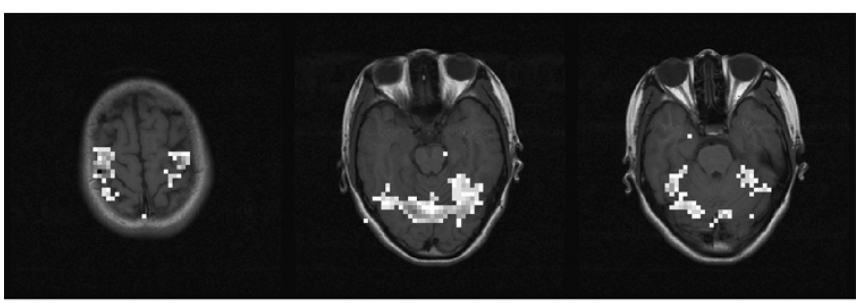

(b)

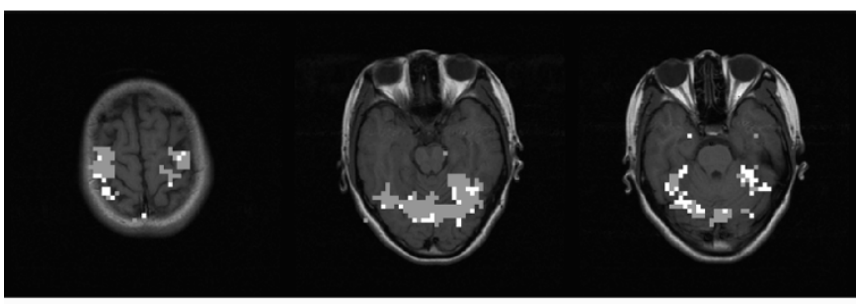

(c)

Fig. 6. Results of comparison for in vivo fMRI data from a PD task (activation maps.) (a) Activation maps of the deconvolution method $\left(p<10^{-7}\right)$. (b) Activation maps of our method $\left(p<10^{-7}\right)$. (c) The difference maps. Light grey areas denote that the F-statistics of the deconvolution method are greater than our method, and white areas denote that the F-statistics of our method are greater than the deconvolution method.

maps, we leave detailed interpretation of the results as a subject for future studies.

Fig. 5 indicates that the F-statistics of the proposed method and the T-statistics of the GLM method are different. Compared with the GLM method, the activated areas revealed by the proposed method are more continuous. In addition, small activated clusters scattered throughout the brain are fewer in the result obtained with the proposed method than that obtained with the GLM method.

Fig. 6 indicates that the results of our method and the deconvolution method are in general agreement and the deconvolution method is slightly more sensitive than the proposed method. However, the F-statistics of the proposed method and the deconvolution method are different. The difference map is presented in Fig. 6(c). Light grey areas denote that the F-statistics of the deconvolution method are greater than our method, and the white areas denote that the F-statistics of our method are greater than the deconvolution method. In addition, a t-test is performed to determine whether the F-statistics $\left(p<10^{-7}\right)$ of the deconvolution and our method have significant differences (in order to perform the t-test, F-statistics are first transformed to Z-statistics with a standard normal distribution). The significance ( $p$-value associated with the T-statistic) is 0.08 . This indicates that F-statistics $\left(p<10^{-7}\right)$ of the deconvolution and our method are significantly different.

To further clarify the rationale of our method, we examine a specific time series corresponding to a highly activated voxel detected by the GLM, the deconvolution and our method. In Fig. 7, the dotted line is the raw time series. The line represents the estimates of the responses of the GLM method, and the corresponding $\mathrm{T}$ statistics is 1.84 . The dashed-dotted line represents the estimates of the responses of the deconvolution method, and the corresponding F statistics is 16.9. The solid line represents the estimates of the responses of our method, and the corresponding F statistics is 18.5. This indicates that, in some voxels, the estimated responses can be slightly improved by our method.

\section{DISCUSSION AND CONCLUSION}

We have proposed a new approach for the analysis of fMRI data. The central idea behind this method is to model the trial-to-trial variability in event-related experiment as meaningful signal. From the viewpoint of methodology, the deconvolution method is a special case of our method when the $\mathbf{H}_{\mathbf{j}}$ s are the same in (8). Compared with the deconvolution method, our method has two obvious advantages: 1) the trial-to-trial variability is modeled as meaningful signal rather than assuming that the HRFs are constant across task events; 2) it is a constrained optimization with an open framework. By including more prior information about the trial or the HRF, it could be enhanced and extended.

In fact, from the viewpoint of statistics, (4) is actually a GLM. However, from the viewpoint of design matrix derivation of the GLM in SPM [11], the meanings of matrix $X$ and $\beta$ in (4) are slightly different from the GLM in SPM. In the GLM of SPM, the design matrix $X$ is specified based on the hemodynamics assumptions, for example a canonical HRF, temporal derivative and dispersion derivative. In contrast, in (4), design matrix $X$ is derived from the stimulation paradigm and polynomial trend model. It can be specified without assumptions concerning the hemodynamics. Here, it is worth noting that the proposed method makes assumptions about the trial-to-trial change in the HRF (i.e., the constraints in the constrained optimization). In addition, in the GLM of SPM, the parameter vector $\beta$ is the regression coefficients that denote the size of the HR. By contrast, in (4), the first $P$ elements in $\beta$ are the estimated HR function.

In (4), modeling the components of the error vector $\varphi$ as independent and normally distributed with mean 0 and variance $\sigma^{2}$ is a simplification. In fact, for example, (4) can be generalized so that it can be applied to data with a stationary and known autocorrelation [26], [27]

$$
K Z=G \beta+K \varphi
$$

where $K$ is a convolution matrix using a Gaussian kernel, $K Z$ represents temporally smoothed data and $G=K X$. The optimum estimator $\beta$ can be obtained by unsmoothing the data by multiplying by $K^{-1}$ and applying least-squares to the smoothed data $K Z$

$$
\hat{\beta}-\left(G^{T} G\right)^{-1} G^{T} K Z
$$

In practice, (5) can be replaced by (16) to model autocorrelation in the presented methods. In addition, there are other 


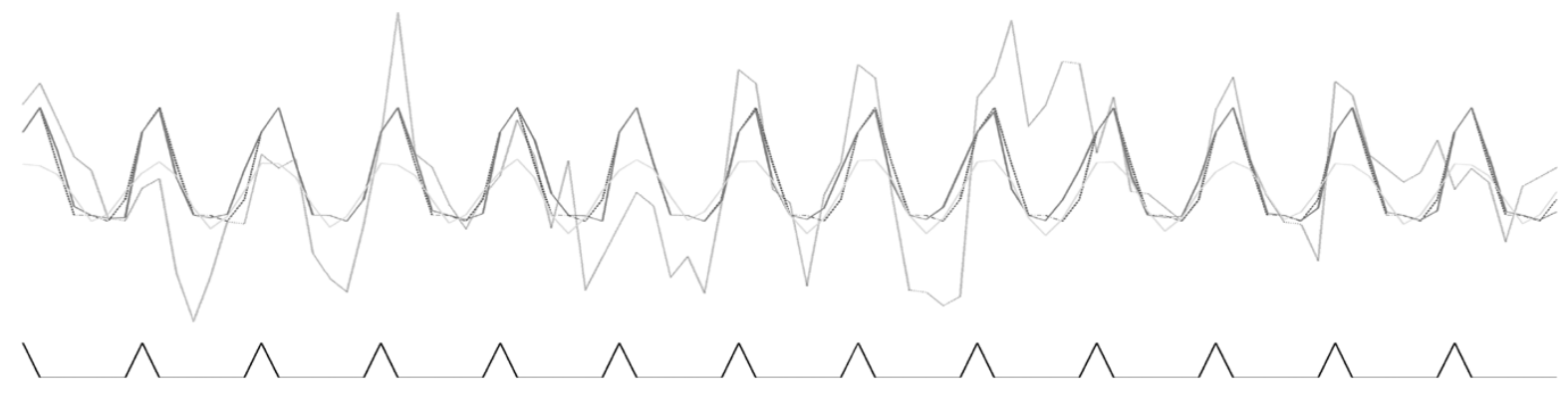

Fig. 7. The time series comes from a highly activated voxel detected by both the deconvolution and our method. The dotted line is the raw time series. The solid line represents the estimates of responses of the GLM method. The dashed-dotted line represents the estimates of responses of the deconvolution method. The solid line represents the estimates of responses of our method, and the spikes at the bottom indicate when the stimuli were presented.

methods can be utilized to model the temporal correlation structure of the noise $\varphi$. For instance, a first order autoregressive model [28], its extension to higher order autoregressive models( in which the autocorrelation structure can including exponential decay and oscillatory terms), and Purdon's [29] method (in which, by adding a second independent white noise term, one can consider the white noise from the scanner).

In this paper, we utilized a simple method to deconvolve the initial HRF for each voxel. However, this method is based on the full-rank of the design matrix $X$ and may show distortions with noisy data. A feasible scheme is to utilize the empirical Bayesian deconvolution formulation, which allows for the specification of the prior of the frequency structure of the fMRI data, to generate the well-constrained priors on the design matrix [30].

We have mentioned that GLM can investigate the trial to trial variability by including parametric modulation. Compared with the parametric modulation in GLM, there are three main differences: 1) Parametric modulation is implemented by a polynomial expansion under the framework of GLM

$$
p(x)=p_{1} x^{n}+p_{2} x^{n-1}+\cdots+p_{n} x+p_{n+1} .
$$

It is a linear combination of basis functions of the experimental parameter. By using nonlinear functions of the task parameter, one can model responses as a nonlinear function of the experimental parameters. As for our method, the trial-to-trial variability is implemented under the constrained optimization framework (12); 2) The basis functions modulated in the parametric modulation method are defined a priori In contrast, in our method, the HRF is calculated by the deconvolution procedure; 3) The relation $\operatorname{FWHM}\left(\mathbf{H}_{\mathbf{i}}\right): \operatorname{FWHM}\left(\mathbf{H}_{\mathbf{j}}\right)=\mathrm{RT}_{i}: \mathrm{RT}_{j}$, $i \neq j$ cannot be modeled by the polynomial expansion. This is the reason that we use the height (rather than width) of the RT to be a function of the trial in the GLM model.

In (12), the first constraint $\mathbf{H}_{\mathbf{j}} \in N(\mathbf{h}, \varepsilon)$ is to assure the physiological meaning of solutions $\mathbf{H}_{\mathbf{j}}$, the second constraint

$$
\operatorname{FWHM}\left(\mathbf{H}_{\mathbf{i}}\right): \operatorname{FWHM}\left(\mathbf{H}_{\mathbf{j}}\right)=\mathrm{RT}_{i}: \mathrm{RT}_{j}, \quad i \neq j
$$

is to model the trial-to-trial variability by RT of different trials. Then, a question rises immediately: which one results in improved performance? In order to answer this question, we compute the area under the ROC curves under three conditions on simulated data sets.
1) With only the first constraint

$$
\begin{array}{ll}
\min _{\mathbf{H}_{\mathbf{j}}} & \left\|\hat{Z}-\sum_{j=1}^{J} \mathbf{H}_{\mathbf{j}} \otimes f_{j}\right\|_{2}^{2} \\
\text { s.t. } & \mathbf{H}_{\mathbf{j}} \in N(\mathbf{h}, \varepsilon) .
\end{array}
$$

2) With only the second constraint

$$
\begin{array}{ll}
\min _{\mathbf{H}_{\mathbf{j}}} & \left\|\hat{Z}-\sum_{j=1}^{J} \mathbf{H}_{\mathbf{j}} \otimes f_{j}\right\|_{2}^{2} \\
\text { s.t. } & \operatorname{FWHM}\left(\mathbf{H}_{\mathbf{i}}\right): \operatorname{FWHM}\left(\mathbf{H}_{\mathbf{j}}\right)=\mathrm{RT}_{i}: \mathrm{RT}_{j}, \quad i \neq j .
\end{array}
$$

3) With both constraints

$$
\begin{aligned}
\min _{\mathbf{H}_{\mathbf{j}}} & \left\|\hat{Z}-\sum_{j=1}^{J} \mathbf{H}_{\mathbf{j}} \otimes f_{j}\right\|_{2}^{2} \\
\text { s.t. } & \mathbf{H}_{\mathbf{j}} \in N(\mathbf{h}, \varepsilon) \\
& \operatorname{FWHM}\left(\mathbf{H}_{\mathbf{i}}\right): \operatorname{FWHM}\left(\mathbf{H}_{\mathbf{j}}\right)=\mathrm{RT}_{i}: \mathrm{RT}_{j}, \\
& i \neq j .
\end{aligned}
$$

The result is shown in Fig. 8. In looking at Fig. 8, we can conclude that: the improved performance mainly comes from the second constraint.

A limitation of the algorithm presented in this paper is that the parameter $\varepsilon$ in the neighborhood $N(\mathbf{h}, \varepsilon)$ was selected empirically. Obviously, the choice of $\varepsilon$ affects the feasible region of (9), and consequently changes the activation map. In theory, increasing $\varepsilon$ in $N(\mathbf{h}, \varepsilon): 1$ ) increases the F-statistics, 2) increases the computational complexity of the method. However, in practice, we find that for different $\varepsilon \mathrm{s}$ the changes of F-statistics are tiny. In order to demonstrate this phenomenon, a one-way ANOVA was performed to determine whether the F-statistics $\left(p<10^{-7}\right)$ for different $\varepsilon$ have significant differences. The $\varepsilon$ values from 0.5 to 5 with an interval of 0.5 were included. The detailed results of ANOVA are listed in Table I. The small F value of 0.02 indicates that the differences between the F-statistics of corresponding $\varepsilon s$ are not significant. The possible reason for this phenomenon may be that the optimum solutions $\mathbf{H}_{\mathbf{j}} s$ are very near to $\mathbf{h}$. Future effort will focus on estimation of the parameter $\varepsilon$ from the measured time series and theoretical investigation of the new formulation of $N(\mathbf{h}, \varepsilon)$ in neighborhood selection. 


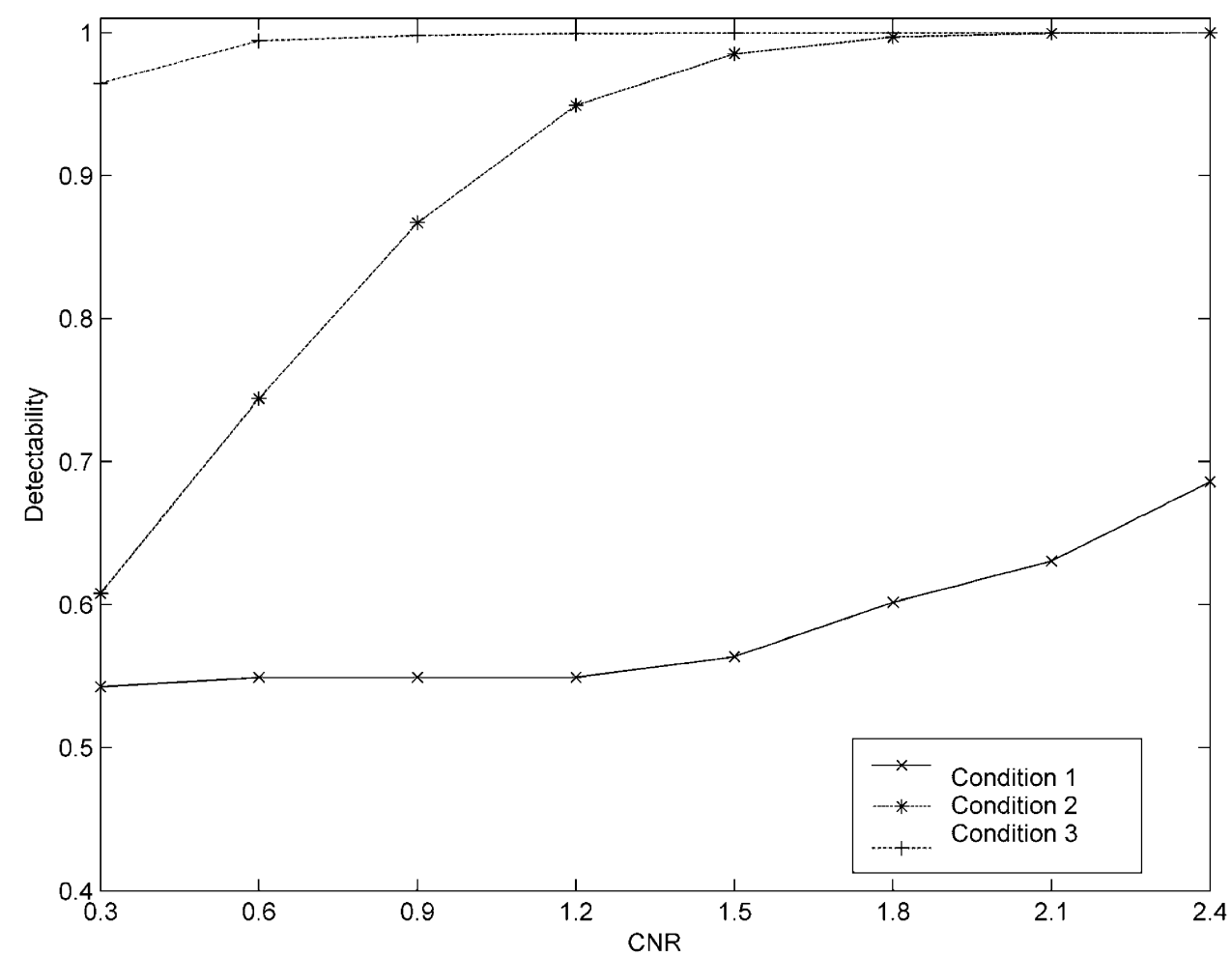

Fig. 8. Performance of condition 1, condition 2, and condition 3 for the simulated data.

TABLE I

ANOVA

\begin{tabular}{lllll}
\hline Source of variation & Sum of Squares & Degrees of Freedom & Mean Squares & F \\
\hline Colums & 0.14 & 9 & 0.01608 & 0.02 \\
Errors & 2230.03 & 2370 & 0.94094 & \\
Total & 2230.18 & 2379 & & \\
\hline
\end{tabular}

On a more critical note, the computation burden required to optimize (12) is an order of magnitude greater than for the deconvolution method, taking several minutes per slice instead of several seconds. This appears to be the price we pay for more utilization of prior information of fMRI time series.

In summary, a new model has been introduced for fMRI data analysis. ROC analysis on simulated data has demonstrated its sensitivity and effectiveness for activation detection. In addition, its usefulness has been demonstrated by applying it to an in vivo event-related dataset. The model can serve as a new model-driven general framework suited to the nature of fMRI data analysis.

\section{ACKNOWLEDGMENT}

The authors are grateful to the anonymous referees for their significant and constructive comments and suggestions, which greatly improved the paper. Y. Lu would also like thank his colleague, Dr. A. Bagshaw, Montreal Neurological Institute, McGill University, Canada, for his carefully checking of the final version of manuscript. The authors give thanks to Prof. W. Xuchu for the kind permission to use the event-related datasets.

\section{REFERENCES}

[1] R. Baumgartner, G. Scarth, C. Teichtmeister, R. Somorjai, and E. Moser, "Fuzzy clustering of gradient-echo functional MRI in the human visual cortex. Part I: Reproducibility,” J. Magn. Reson. Imag., vol. 7, pp. 1094-1101, 1997.

[2] P. Filzmoser, R. Baumgartner, and E. Moser, "A hierarchical clustering method for analyzing functional MR images," Magn. Reson. Imag., vol. 17, pp. 817-826, 1999.

[3] C. Goutte, P. Toft, E. Rostrup, F. A. Nielsen, and L. K. Hansen, "On clustering fMRI time series," Neurolmage, vol. 9, no. 3, pp. 298-310, 1999.

[4] G. Scarth, R. Somorjai, M. Alexander, B. Wowk, A. Wennerberg, and M. McIntyre, "Cluster analysis of functional brain images," in Human Brain Mapping. New Yourk: Wiley, 1995, vol. S1, Proceedings of the First International Conference on Functional Mapping of the Human Brain, pp. $158-158$.

[5] L. K. Hansen et al., "Generalizable patterns in neuroimaging: How many principal components," NeuroImage, vol. 9, no. 5, pp. 534-544, 1999.

[6] S. H. Lai and M. Fang, "A novel local PCA-based method for detecting activation signals in fMRI," Magn. Reson. Imag., vol. 17, no. 6, pp. 827-836, 1999.

[7] V. D. Calhoun, T. Adali, G. D. Pearlson, and J. J. Pekar, "Spatial and temporal independent component analysis of functional MRI data containing a pair of task-related waveforms," Human Brain Mapping, vol. 13, no. 1, pp. 43-53, 2001.

[8] T. P. Jung, S. Makeig, M. J. Mckeown, A. J. Bell, T. W. Lee, and T. J. Sejnowski, "Imaging brain dynamics using independent component analysis," Proc. IEEE, vol. 89, no. 7, pp. 1107-1122, Jul. 2001. 
[9] M. J. McKeown, S. Makeig, G. G. Brown, T. P. Jung, S. S. Kindermann, A. J. Bell, and T. J. Sejnowski, "Analysis of fMRI data by blind separation into independent spatial components," Human Brain Mapping, vol. 6, no. 3, pp. 160-188, 1998.

[10] S. C. Ngan and X. Hu, "Analysis of functional magnetic resonance imaging data using self-organizing mapping with spatial connectivity," Magn. Reson. Med., vol. 41, no. 5, pp. 939-946, 1999.

[11] K. J. Friston, A. P. Holmes, K. J. Worsley, J. P. Poline, C. D. Frith, and R. S. J. Frackowiak, "Statistical parametric maps in functional imaging: A general linear approach," Human Brain Mapping, vol. 2, pp. 189-210, 1995.

[12] G. H. Glover, "Deconvolution of impulse response in event-related BOLD fMRI," NeuroImage, vol. 9, no. 4, pp. 416-429, 1999.

[13] B. D. Ward. (2002, May) Deconvolution Analysis of fMRI Time Series Data. [Online]. Available: http://www.afni.nimh.gov/afni/edu

[14] G. K. Aguirre, E. Zarahn, and M. D'Esposito, "The variability of human, BOLD hemodynamic responses," NeuroImage, vol. 8, no. 4, pp. 360-369, 1998.

[15] J. R. Duann, T. P. Jung, W. J. Kuo, T. C. Yeh, S. Makeig, J. C. Hsieh, and J. T. Sejnowski, "Single-trial variability in event-related BOLD signals," Neurolmage, vol. 15, no. 4, pp. 823-835, 2002.

[16] W. Richter, K. Ugurbil, A. Georgopoulos, and S. G. Kim, “Time-resolved fMRI of mentalrotation," NeuroReport, vol. 8, no. 17, pp. 3697-3702, 1997

[17] F. W. Gembicki, "Vector optimization for control with performance and parameter sensitivity indices," Ph.D. dissertation, Case Western Reserve Univ, Cleveland, OH, 1974.

[18] C. Buchel, R. J. S. Wise, C. J. Mummery, J. B. Poline, and K. J. Friston, "Nonlinear regression in parametric activation studies," NeuroImage, vol. 4, no. 1, pp. 60-66, 1996.

[19] C. Buchel, A. P. Holmes, G. Rees, and K. J. Friston, "Characterizing stimulus-response functions using nonlinear regressors in parametric fMRI experiments," NeuroImage, vol. 8, no. 2, pp. 140-148, 1998.
[20] K. J. Friston, P. Fletcher, O. Josephs, A. Holmes, M. D. Rugg, and R. Turner, "Event-related fMRI: Characterizing differential responses," NeuroImage, vol. 7, no. 1, pp. 30-40, 1998.

[21] N. Lange, "Tutorial in biostatistics: Statistical approaches to human brain mapping by functional magnetic resonance imaging," Statist. Med., vol. 15, pp. 389-428, 1996.

[22] P. A. Bandettini, E. C. Wong, R. S. Hinks, R. S. Tikofsky, and J. S Hyde, "Time course EPI of human brain function during task activation," Magn. Reson. Med., vol. 25, no. 2, pp. 390-397, 1992.

[23] M. J. Fadili, S. Ruan, D. Bloyet, and B. Mazoyer, "A multistep unsupervised fuzzy clustering analysis of fMRI time series," Human Brain Mapping, vol. 10, no. 4, pp. 160-178, 2000.

[24] K. K. Kwong et al., "Dynamic magnetic resonance imaging of human brain activity during primary sensory stimulation," Proc. Nat. Acad. Sci., vol. 89, pp. 5675-5679, 1992.

[25] P. A. Bandettini, A. Jesmanowicz, E. C. Wong, and J. S. Hyde, "Processing strategies for time course data sets in functional MRI of the human brain," Magn. Reson. Med., vol. 30, no. 2, pp. 161-173, 1993.

[26] K. J. Friston et al., "Analysis of fMRI time-series revisited," $\mathrm{Neu}$ rolmage, vol. 2, no. 3, pp. 173-81, 1995.

[27] K. J. Worsley and K. J. Friston, "Analyisis of fMRI time-series revisitedagain," NeuroImage, vol. 2, pp. 173-181, 1995

[28] E. T. Bullmore et al., "Statistical methods of estimation and inference for functional MR image analysis," Magn. Reson. Med., vol. 35, pp. 261-277, 1996.

[29] P. L. Purdon, V. Solo, R. M. Weisskoff, and E. Brown, "Locally regularized spatiotemporal modeling and model comparison for functional MRI," NeuroImage, vol. 14, no. 4, pp. 912-923, 2001.

[30] D. R. Gitelman, W. D. Penny, J. Ashburner, and K. J. Friston, "Modeling regional and psychophysiologic interactions in fMRI: The importance of hemodynamic deconvolution," NeuroImage, vol. 19, no. 1, pp. 200-207, 2003. 